Proceedings of the ISCIE International Symposium on Stochastic Systems Theory and Its Applications Osaka, Nov. 10-12, 1993

\title{
ON THE ANALYTICAL AND NUMERICAL STUDY OF SEARCHLIGHT PROBLEM IN TURBID SLAB
}

\author{
S. Ueno ${ }^{1}$, M. Toho ${ }^{1}$ and A.P. Wang ${ }^{2}$
}

1)Information Science Laboratory, Kyoto School of Computer Science, Kyoto 606, Japan, 2)Department of Mathematics, Arizona State University, Tempe, Arizona 85287, USA

\begin{abstract}
In the present paper, it is discussed how to treat with the analytical and numerical solutions of searchlight problem with faint background light on flat terrain bounded below by a diffuse reflector. It is elucidated that the angular distribution of radiation emergent from the top of the turbid slab of finite thickness with a point source on top is discussed by imbedding the problem within a family of parameters, allowing for the numerical solution via Monte Carlo method.
\end{abstract}

\section{INTRODUCTION}

In this paper we discuss the illumination by a narrow collimated beam such as a searchlight. The turbid slab in the vicinity of the beam is assumed as plane-parallel, being bounded below by reflective surface. In other words, the searchlight problem under consideration concerns the reflection of a narrow pencil of radiation of infinitesimal cross section, but of finite irradiation, on the top of a vertically homogeneous and anisotropically scattering slab bounded below by the horizontally homogeneous albedo of the surface. The main interest is for example in such backward scattered light as in lidar remote sensing (cf. [1] - [17]).

In this paper it is stated how to obtain analytically the Cauchy system of the scattering function in searchlight problem in turbid slab bounded by a diffuse reflector. An invariant imbedding in the initial-value solution of the scattering function for searchlight results in the Riccati-type of the non-linear integrodifferential equation with the initial condition.

\section{BASIC EQUATIONS}

In this section, based on a three-dimensional radiative transfer theory, we treat the searchlight problem in turbid slab bounded by a flat reflecting surface with horizontally uniform albedo.

The equation of transfer appropriate to the present case takes the form

$$
\Omega \cdot \nabla I(z, x, y ; \Omega)+\alpha I=\frac{\sigma}{4 \pi} \int_{4 \pi} P\left(z ; \Omega, \Omega^{\prime}\right) I\left(z, x, y ; \Omega^{\prime}\right) d \Omega^{\prime}
$$

where $\Omega \cdot \nabla I$ is the directional derivative of the intensity in Cartesian coordinates, $\alpha$ the attenuation coefficient, $\sigma$ the scattering coefficient, $P$ the depth-dependent phase function, $-\infty \leq x, y \leq \infty$, and $d \Omega^{\prime}=d v^{\prime} d \phi^{\prime}$, where $v$ is the cosine of zenith distance and $\phi$ is the azimuth. Eq.(1) should be solved subject to the boundary conditions

$$
I\left(z_{1}, x, y ;-\Omega\right)=\pi\left[F \delta\left(\Omega-\Omega_{0}\right) \delta(x) \delta(y)\right]
$$

and

$$
I(0, x, y ; \Omega)=\frac{1}{v} \int_{2 \pi} k\left(\Omega, \Omega^{\prime}\right) I\left(0, x, y ;-\Omega^{\prime}\right) v^{\prime} d \Omega^{\prime}
$$

where $\delta\left(\Omega-\Omega_{0}\right)=\delta(v-u) \delta\left(\phi-\phi_{0}\right)$ is the Dirac delta function and $d x=\left(x-x_{0}\right), d y=\left(y-y_{0}\right)$. In Eq. (3) the bidirectional reflection law $k\left(\Omega, \Omega^{\prime}\right)$ represents the probability that a photon incident on the bottom $(0, x, y)$ in the downward direction $\Omega^{\prime}$ will be reflected from it in the upward direction $\Omega$ within an elementary solid angle. In the case of isotropic reflection we put

$$
k\left(x, y ; \Omega, \Omega^{\prime}\right)=\frac{v A(x, y)}{\pi},
$$


where $A(x, y)$ is the horizontally inhomogeneous albedo of the reflecting surface; e.g., in accordance with Lambert's law. It represents the ratio of the total energy reflected by the surface to the incident energy. In this case Eq.(3) becomes

$$
I(0, x, y ; \Omega)=\frac{A(x, y)}{\pi} \int_{2 \pi} I\left(0, x, y ;-\Omega^{\prime}\right) v^{\prime} d \Omega^{\prime} .
$$

In Eq.(5) $I\left(0, x, y ;-\Omega^{\prime}\right)$ consists of the downwards directly transmitted intensity and the downwards diffusely transmitted intensity as below

$$
I\left(0, x, y ;-\Omega^{\prime}\right)=\pi \delta\left(\Omega-\Omega^{\prime}\right)[F \delta(d x) \delta(d y)] e^{-\tau / v^{\prime}}+I^{*}\left(0, x, y ;-\Omega^{\prime}\right) .
$$

In Eq.(6) $\tau$ denotes the total optical thickness, and I* represents the diffuse radiation field under consideration. The definition of the optical thickness of the atmosphere $\tau$ is given by

$$
\tau=\alpha z_{1} .
$$

\section{CAUCHY SYSTEM OF SCATTERING FUNCTION}

The diffusely reflected intensity $I\left(z_{1}, x, y ;+v, \phi\right)$ due to a pencil of incident radiation takes the form

$$
\begin{aligned}
& I\left(z_{1}, x, y ;+v, \phi\right)=I\left(z_{1}, x, y ;+v, \phi ; I_{\text {inc }}\right)= \\
& \quad=\frac{1}{2 \pi} \int_{-\infty}^{\infty} \int_{-\infty}^{\infty} \int_{0}^{1} \int_{0}^{2 \pi} R\left(z_{1}, x-x^{\prime}, y-y^{\prime} ; v, \phi ; v^{\prime}, \phi^{\prime}\right) I_{i n c}\left(x^{\prime}, y^{\prime} ;-v^{\prime}, \phi^{\prime}\right) d x^{\prime} d y^{\prime} d v^{\prime} d \phi^{\prime} \\
& \quad=\frac{F}{2} R\left(z_{1}, x, y ; v, \phi ; u, \phi_{0}\right),
\end{aligned}
$$

where $I_{\text {inc }}(x, y ;-v, \phi)$ is equal to $I\left(z_{1}, x, y ;-v, \phi\right)$ in Eq.(2). For the sake of simplicity the point of incidence is assumed to coincide with the origin.

The addition of a layer of infinitesimally small geometrical thickness $\Delta z$ to the upper boundary $z=z_{1}$ gives rise to the diffusely reflected light in the direction $(+v, \phi)$ at the point $\left(z_{1}+\Delta z, x+\right.$ $\Delta z \tan \theta \cos \phi, y+\Delta z \tan \theta \sin \phi)$ given by

$$
\begin{aligned}
I\left(z_{1}\right. & \left.+\Delta z, x+\Delta z \tan \theta \cos \phi, y+\Delta z \tan \theta \sin \phi ;+v, \phi ; I_{i n c}^{*}\right) \\
& =\frac{1}{2 \pi} \int_{-\infty}^{\infty} \int_{-\infty}^{\infty} \int_{0}^{1} \int_{0}^{2 \pi} R\left(z_{1}+\Delta z, x+\Delta z \tan \theta \cos \phi-x^{\prime}, y+\Delta z \tan \theta \sin \phi-y^{\prime} ;\right. \\
& \left.v, \phi ; v^{\prime}, \phi^{\prime}\right) I_{i n c}^{*}\left(x^{\prime}, y^{\prime} ;-v^{\prime}, \phi^{\prime}\right) d x^{\prime} d y^{\prime} d v^{\prime} d \phi^{\prime} \\
& =\frac{F}{2} R\left(z_{1}+\Delta z, x+\Delta z\left(\tan \theta \cos \phi+\tan \theta_{0} \cos \phi_{0}\right),\right. \\
& \left.y+\Delta z\left(\tan \theta \sin \phi+\tan \theta_{0} \sin \phi_{0}\right) ; v, \phi ; u, \phi_{0}\right),
\end{aligned}
$$

where

$$
I_{i n c}^{*}(x, y ;-v, \phi)=\pi F \delta\left(x+\Delta z \tan \theta_{0} \cos \phi_{0}\right) \delta\left(y+\Delta z \tan \theta_{0} \sin \phi_{0}\right) \delta(v-u) \delta\left(\phi-\phi_{0}\right) .
$$

Following the procedure of an unified approach [11], we shall apply an invariant imbedding argument to $I\left(z_{1}, x, y ;+v, \phi ; I_{\text {inc }}\right)$ as below

$$
\begin{array}{r}
I\left(z_{1}+\Delta z, x+\Delta z \tan \theta \cos \phi, y+\Delta z \tan \theta \sin \phi ;+v, \phi ; I_{i n c}^{*}\right)= \\
=I\left(z_{1}, x, y ;+v, \phi ; \hat{I}_{i n c}\right)+\Delta z \cdot G\left(z_{1}, x, y ;+v, \phi\right)+\theta(\Delta z),
\end{array}
$$

where $\hat{I}_{\text {inc }}$ is the modified intensity of radiation incident on the atmosphere when the layer of the geometrical thickness $\Delta z$ is added to the upper boundary and $\theta(\Delta z)$ is of the order of magnitude of $(\Delta z)^{2}$. 
From the transfer equation in downwards direction, we have

$$
\begin{aligned}
& \hat{I}_{\text {inc }}(x, y ;-v, \phi)= \\
& \quad=\pi F \delta(x) \delta(y) \delta(v-u) \delta\left(\phi-\phi_{0}\right)\left(1-\alpha\left(z_{1}\right) \frac{\Delta z}{v}\right) \\
& \quad+\frac{\sigma\left(z_{1}\right)}{4 \pi} \frac{\Delta z}{v}\left[\pi F P\left(z_{1} ;-v, \phi ;-u, \phi_{0}\right) \delta(x) \delta(y)\right. \\
& \left.\quad+\frac{F}{2} \int_{0}^{1} \int_{0}^{2 \pi} P\left(z_{1} ; v, \phi ;-v^{\prime}, \phi^{\prime}\right) R\left(z_{1}, x, y ; v^{\prime}, \phi^{\prime} ; u, \phi_{0}\right) d v^{\prime} d \phi^{\prime}\right] .
\end{aligned}
$$

The substitution of Eq.(12) into the first term on the right-hand side of Eq.(11) yields

$$
\begin{aligned}
I\left(z_{1}, x, y ;+v, \phi ; \hat{I}_{i n c}\right) & \\
\quad= & \frac{1}{2 \pi} \int_{-\infty}^{\infty} \int_{-\infty}^{\infty} \int_{0}^{1} \int_{0}^{2 \pi} R\left(z_{1}, x-x^{\prime}, y-y^{\prime} ; v, \phi ; v^{\prime}, \phi^{\prime}\right) \hat{I}_{i n c}\left(x^{\prime}, y^{\prime} ;-v^{\prime}, \phi^{\prime}\right) d x^{\prime} d y^{\prime} d v^{\prime} d \phi^{\prime} \\
\quad & \frac{F}{2} R\left(z_{1}, x, y ; v, \phi ; u, \phi_{0}\right)\left(1-\alpha\left(z_{1}\right) \frac{\Delta z}{u}\right) \\
& +\frac{\sigma\left(z_{1}\right)}{4 \pi} \Delta z \frac{F}{2}\left[\int_{0}^{1} \int_{0}^{2 \pi} R\left(z_{1}, x, y ; v, \phi ; v^{\prime}, \phi^{\prime}\right) P\left(z_{1} ;-v^{\prime}, \phi^{\prime} ;-u, \phi_{0}\right) \frac{d v^{\prime}}{v^{\prime}} \phi^{\prime}\right. \\
& +\frac{1}{2 \pi} \int_{-\infty}^{\infty} \int_{-\infty}^{\infty} \int_{0}^{1} \int_{0}^{2 \pi} \int_{0}^{1} \int_{0}^{2 \pi} R\left(z_{1}, x-x^{\prime}, y-y^{\prime} ; v, \phi ; v^{\prime}, \phi^{\prime}\right) \\
& \left.\times P\left(z_{1} ;-v^{\prime}, \phi^{\prime} ; v^{\prime \prime}, \phi^{\prime \prime}\right) R\left(z_{1}, x^{\prime}, y^{\prime} ; v^{\prime \prime}, \phi^{\prime \prime} ; u, \phi_{0}\right) d x^{\prime} d y^{\prime} \frac{d v^{\prime}}{v^{\prime}} d \phi^{\prime} \frac{d v^{\prime \prime}}{v^{\prime \prime}} d \phi^{\prime \prime}\right] .
\end{aligned}
$$

On making use of Eq.(6), we get

$$
\begin{aligned}
& G\left(z_{1}, x, y ;+v, \phi\right)=-\frac{F}{2 v}\left[\alpha\left(z_{1}\right) R\left(z_{1}, x, y ; v, \phi ; u, \phi_{0}\right)\right. \\
& \quad-\frac{\sigma\left(z_{1}\right)}{4 \pi} \int_{0}^{1} \int_{0}^{2 \pi} P\left(z_{1} ; v, \phi ; v^{\prime}, \phi^{\prime}\right) R\left(z_{1}, x, y ; v^{\prime}, \phi^{\prime} ; u, \phi_{0}\right) d v^{\prime} d \phi^{\prime} \\
& \left.\quad-\frac{\sigma\left(z_{1}\right)}{4 \pi} 2 \pi \delta(x) \int_{0}^{1} \int_{0}^{2 \pi} P\left(z_{1} ; v, \phi ;-v^{\prime}, \phi^{\prime}\right) \delta\left(v^{\prime}-u\right) \delta\left(\phi^{\prime}-\phi_{0}\right) d v^{\prime} d \phi^{\prime}\right]
\end{aligned}
$$

Then, combining Eqs.(11), (13), (14), we have

$$
\begin{aligned}
R\left(z_{1}\right. & +\Delta z, x+\Delta z\left(\tan \theta \cos \phi+\tan \theta_{0} \cos \phi_{0}\right), y+\Delta z\left(\tan \theta \sin \phi+\tan \theta_{0} \sin \phi_{0} ; v, \phi ; u, \phi_{0}\right) \\
& =R\left(z_{1}, x ; v, \phi ; u, \phi_{0}\right)-\alpha\left(z_{1}\right) \frac{\Delta z}{u} R\left(z_{1}, x, y ; v, \phi ; u, \phi_{0}\right) \\
& +\frac{\sigma\left(z_{1}\right)}{4 \pi} \Delta z\left[\int_{0}^{1} \int_{0}^{2 \pi} R\left(z_{1}, x, y ; v, \phi ; v^{\prime}, \phi^{\prime}\right) P\left(z_{1} ;-v^{\prime}, \phi^{\prime} ;-u, \phi_{0}\right) \frac{d v^{\prime}}{v^{\prime}} d \phi^{\prime}\right. \\
& +\frac{1}{2 \pi} \int_{-\infty}^{\infty} \int_{-\infty}^{\infty} \int_{0}^{1} \int_{0}^{2 \pi} \int_{0}^{1} \int_{0}^{2 \pi} R\left(z_{1}, x-x^{\prime}, y-y^{\prime} ; v, \phi ; v^{\prime}, \phi^{\prime}\right) P\left(z_{1} ;-v^{\prime}, \phi^{\prime} ; v^{\prime \prime}, \phi^{\prime \prime}\right) \\
& \times R\left(z_{1}, x^{\prime}, y^{\prime} ; v^{\prime \prime}, \phi^{\prime \prime} ; u, \phi_{0}\right) d x^{\prime} d y^{\prime} \frac{d v^{\prime}}{v^{\prime}} d \phi^{\prime} d v^{\prime \prime} d \phi^{\prime \prime} \\
& +\frac{1}{v} \int_{0}^{1} \int_{0}^{2 \pi} P\left(z_{1} ; v, \phi ; v^{\prime}, \phi^{\prime}\right) R\left(z_{1}, x, y ; v^{\prime}, \phi^{\prime} ; u, \phi_{0}\right) d v^{\prime} d \phi^{\prime} \\
& \left.+2 \pi \frac{\delta(x) \delta(y)}{v} P\left(z_{1} ; v, \phi ;-u, \phi_{0}\right)\right]-\alpha\left(z_{1}\right) \frac{\Delta z}{v} R\left(z_{1}, x, y ; v, \phi ; u, \phi_{0}\right) .
\end{aligned}
$$

Putting $\Delta z \rightarrow 0$ in Eq.(15), and writing

$$
R\left(z_{1}, x, y ; v, \phi ; u, \phi_{0}\right)=\frac{1}{2 v} S\left(z_{1}, x, y ; v, \phi ; u, \phi_{0}\right),
$$


we obtain an initial-value solution of the three-dimensional scattering function $S\left(z_{1}, x, y ; \Omega, \Omega_{0}\right)$ fulfilling the following Riccati-type of nonlinear integrodifferential equation :

$$
\begin{aligned}
\frac{\partial S}{\partial z_{1}}\left(z_{1}, x, y ; \Omega, \Omega_{0}\right)+\left(\tan \theta \cos \phi+\tan \theta_{0} \cos \phi_{0}\right) \frac{\partial S}{\partial x}+ \\
\quad+\left(\tan \theta \sin \phi+\tan \theta_{0} \sin \phi_{0}\right) \frac{\partial S}{\partial y}+\alpha\left(\frac{1}{v}+\frac{1}{u}\right) S= \\
=\sigma\left[P\left(\Omega,-\Omega_{0}\right) \delta(x) \delta(y)+\frac{1}{4 \pi} \int_{2 \pi} S\left(z_{1}, x, y ; \Omega, \Omega^{\prime \prime}\right) P\left(-\Omega^{\prime \prime},-\Omega_{0}\right) \frac{d \Omega^{\prime \prime}}{v^{\prime \prime}}+\right. \\
\quad+\frac{1}{4 \pi} \int_{2 \pi} P\left(\Omega, \Omega^{\prime}\right) S\left(z_{1}, x, y ; \Omega^{\prime}, \Omega_{0}\right) \frac{d \Omega^{\prime}}{v^{\prime}}+ \\
\quad+\frac{1}{16 \pi^{2}} \int_{-\infty}^{\infty} \int_{-\infty}^{\infty} \int_{2 \pi} \int_{2 \pi} S\left(z_{1}, x-x^{\prime}, y-y^{\prime} ; \Omega, \Omega^{\prime}\right) \times \\
\left.\quad \times P\left(-\Omega^{\prime}, \Omega^{\prime \prime}\right) S\left(z_{1}, x^{\prime}, y^{\prime} ; \Omega^{\prime \prime}, \Omega_{0}\right) d x^{\prime} d y^{\prime} \frac{d \Omega^{\prime}}{v^{\prime}} \frac{d \Omega^{\prime \prime}}{v^{\prime \prime}}\right]
\end{aligned}
$$

together with the initial condition

$$
S\left(0, x, y ; \Omega, \Omega_{0}\right)=4 A v u \delta(x) \delta(y) .
$$

Furthermore, it should be mentioned that the generalized S-function is global invariant with respect to the angular arguments.

\section{ASYMPTOTIC SOLUTION}

In the case of simultaneous atmospheric illumination by searchlight and faint background light, the relation between the total reflection $R$ and the diffuse reflection operator $\rho$, i.e., a solution of three-dimensional transfer equation in a free space is expressed in terms of the operators $\tilde{t}, r, \tilde{\tau}$ as below.

$$
R(K)=\rho+\tilde{t} K \cdot \sum_{n=0}(r K)^{n} \cdot \tilde{\tau}
$$

where the second term denotes the multiple scattering taking place between the atmospheric layer and earth reflection $K$. The operators $\tilde{t}$ and $\tilde{\tau}$ are the total upward and downward transmission operators in a free space, consisting of the direct transmission and diffuse ones, both of them being one-dimensional and operator $r$ is obtained by solving Chandrasekhar's equation in free space (cf. [1], [3], [5], [12]).

On decomposing the ground reflection $K$ into two parts, i.e., $K_{g}$ the ground reflection and $K_{t}$ the target reflection, i,e.,

$$
K=K(x, y)=K_{g}+K_{t}
$$

we obtain approximately

$$
R(K)=-\rho+R\left(K_{t}\right)+R\left(K_{g}\right)+\tilde{t} K_{t} r K_{g} \tilde{\tau}+\cdots
$$

where $R\left(K_{t}\right)$ is the total reflection due to the atmsopheric layer and target and $R\left(K_{g}\right)$ is due to the atmospheric layer and ground. The remaining term involves higher order interactions between $K_{t}$, $K_{g}$ and atmospheric layer. Under the assumption of small $\left\|K_{g}\right\|$, the following approximation is obtained

$$
R\left(K_{t}\right) \doteq R(K)-R\left(K_{g}\right)+\rho .
$$

If there is prior knowledge of $R\left(K_{g}\right)$ and $\rho$, then measurement of $R(K)$ gives an approximation of $R\left(K_{t}\right)$ by Eq.(22). Thus, under the first approximation without taking any interaction between target and atmospheric scattering, the target reflection $K_{t}$ takes the form

$$
K_{t}^{(1)} \doteq\left[R\left(K_{t}\right)-\rho\right] \exp \left[\frac{2}{v} \int_{0}^{h} \alpha d z\right] \delta\left(\Omega+\Omega_{0}\right)^{-1}
$$




\section{NUMERICAL SIMULATION VIA MONTE CARLO METHOD}

The numerical simulation for the searchlight problem has been carried out under the somewhat restricted initial and boundary conditions via Monte Carlo method. Assume that the atmosphere under consideration is throughout optically uniform, bounded with the horizontally uniform surface of ground. Furthermore, the searchlight is perpendicularly incident on the origin of the Cartesian coordinates.

Under such initial and boundary conditions the numerical simulation of multiple scattering processes of photons is carried out by Monte Carlo method. The free path of photons $(l)$ is calculated using uniformly random number $r_{u}$ by the Eq.(24),

$$
l=C_{r} \frac{\tau}{z_{1}} \log r_{u}
$$

where $C_{r}$ is a parameter which regulates the path length after reflection. In the case of reflection on the flat surface the value of $C_{r}$ is equal to 1.42 , whereas $C_{r}$ is unity after re-emission by the atmospheric particles.

The several simulations were performed and in each case the optical thickness of the atmosphere had a different value between 0.01 and 10.0. It is assumed that the scattering coefficient of atmospheric particles $(\lambda)$ is 1.0; the ground albedo $A$ is 0.4 ; the incident direction of the photons is normal, i.e., $\left(\theta_{0}, \phi_{0}\right)=(0,0)$; the total number of photons is 3.2 millions; the aperture of the photosensor at the top is 0.2 .

Then, the radiation intensity under consideration $I$ for incident flux $F$ at the top is estimated as below.

$$
I\left(z_{1}, 0,0 ; \Omega\right)=\pi F \frac{\Delta N\left(z_{1}, 0,0 ; \Omega\right)}{v N \Delta s},
$$

where $\Delta s$ is the surface area of the aparture at top and $\Delta N$ is the number of photons passed through the aparture in direction $\Omega$. The number of incident photons from the searchlight $N$ is conserved throughout the whole process.

Under such initial and boundary conditions the polar dependence of the reflected searchlight intensity at the origin of top $I\left(z_{1}, 0,0 ; \Omega\right)$ is numerically simulated in Fig.1 and Fig2. It shows that the intensity contours at the origin of top for normal incidence have similar tendency. In other words, from the horizontal direction to the normal ones the radiation intensity increases monotonically, arriving at muximum for normal incidence. As the optical thicknes increases the sharp peak near $v=1\left(\theta_{0}=0\right)$ disappears.

The statistical error in the simulation depends on the number of emitted photons, the optical thickness, the polar angle of reflected photon and the differential coefficient of estimated curves. It can be expected to be in the range between one to ten percent in our calculation.

\section{DISCUSSION}

It is shown how to obtain the Cauchy system of scattering function in searchlight problem via invariant imbedding. The initial-value solution coincides with that obtained via the integral operator method (cf. [7]). The approximate solution of the combined problem in allowance for the searchlight and weak uniform background light is discussed.

A numerical approximation of a solution of Eq.(1) was tried via the Monte Carlo method for the uniform and isotropic atmosphere with a uniform Lambertian reflector, assuming the isotropic scattering. A variable reflection parameter is used to relate our stochastic solution to the theoretical one in the case of parallel rays. The result shows us the angular distribution of the diffusely reflected searchlight intensity. The estimated value should be compared with theoretical or experimental ones.

The error estimation is difficult because of complexity of the system. However, we can treat the error statistically. Effective error estimation will reduce computing time. 


\section{REFERENCES}

[1] S. Chandrasekhar, Radiative Transfer, Cambridge Univ. Press, London, UK, (1950).

[2] S. Chandrasekahr, Proc. Natl. Acad. Sci. U.S.A. Vol.44, 938-942 (1958).

[3] I.W. Busbridge, Astrophys J. Vol.133, 198-209 (1961).

[4] R. Bellman, R. Kalaba and S. Ueno, J. Math. Analys. Appl. Vol.7, 310-321 (1963).

[5] V.V. Sobolev, Light Scattering in Planetary, Atmospheres (Translated by W.M. Irvine), Pergamon Press, Oxford, UK., (1973).

[6] J. Lenoble (Editor), Radiative Transfer in Scattering and Absorbing Atmospheres: Standard Computational Procedures, A. Deepak Publ., Virginia, (1985).

[7] S. Ueno, J. Math. Analys. Appl. Vol.119, 223-233 (1986).

[8] A.P. Wang, Math. Modeling, Vol.9, (2), 117-124 (1987).

[9] A.P. Wang and S. Ueno, J. Math. Phys. Vol.34, pp.878 - 884 (1993).

[10] A.P.Wang, J. Math. Physics, Vol.18, 47-51 (1977).

[11] G.M. Wing, An Introduction to Transport Theory, Wiley, New York, (1962).

[12] S. Ueno, Astrophys. J. Vol.132, 729-745 (1960).

[13] S. Ueno and A.P. Wang. Computers and Mathematics with Applications, Pergamon Press. Vol.21, 1-6 (1991).

[14] S. Ueno and A.P. Wang. In Proc. of IEEE Int. Conf. on System Engineering, Kobe, Japan, Sept.17-19, pp.377-380 (1992).

[15] A.P. Wang and S. Ueno. In Proc. of the Bellman Continuum, in Univ. of Hawaii, Manoa, pp.1-9 (1993). To appear in Computers Math. Applic.

[16] G.I. Marchuk et al., The Monte Carlo Methods in Atmospheric Optics. Springer-Verlag, Berlin, (1980).

[17] S.Ueno, M.Toho and A.P.Wang. To appear in Proc. of the 25th IGARSS'93, Kogakuin University, Tokyo, Japan, Aug.18 - 21, pp.1279 - 1282 (1993).

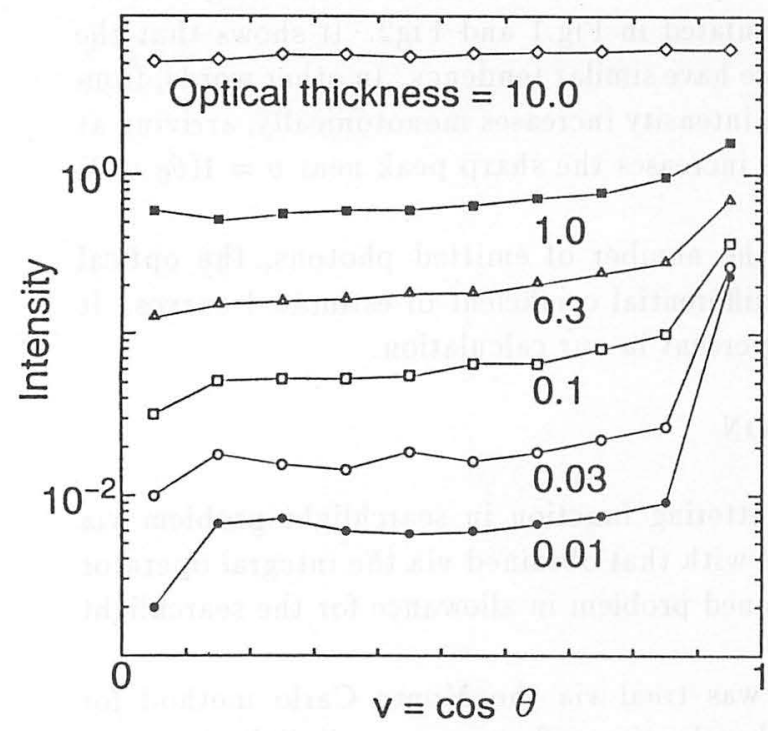

Fig.1 Polar angle dependence of intensity. Case 1: Search light on the top of atmospher

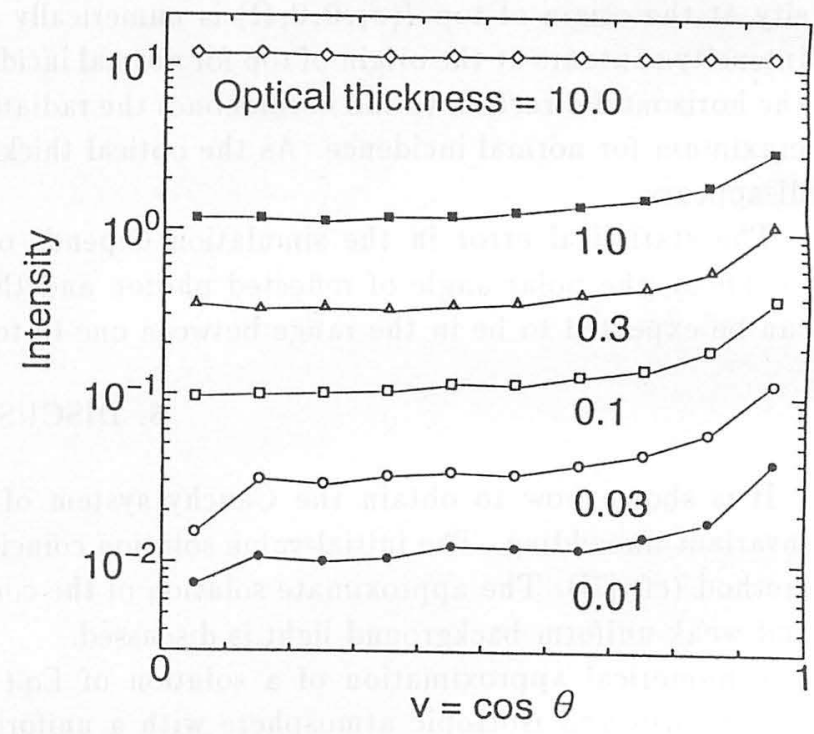

Fig.2 Polar angle dependence of intensity. Case 2: Search light on the bottom of atmospher 